\title{
A Comprehensive Research on Video Imaging Techniques
}

\author{
Farooq Sunar Mahammad, Karthik Balasubramanian, T. Sudhakar Babu, \\ V. Madhu iswanatham
}

\begin{abstract}
In today's world, Video imaging techniques are extremely important due to its manifold advantages as they contribute extensively to connectivity of people from one part of the world to another. In this constrain the authors focused to emphasize the numerous technologies available for video image process, highlights of the significant research works carried out in Video imaging techniques has been represented as a review article. Further the article will give the more information about latest techniques; it also summarizes the features of all works with respect to video imaging processing techniques. Hence, the objective of this work is to benefit scientists and new entrants in the field of Video imaging to get overview of the technologies.

Index Terms-P2P Video Streaming, Real time video streaming, Non-Real time video streaming
\end{abstract}

\section{INTRODUCTION}

In the modern world, engineering innovation has transformed the world to a better place for mankind. There are many inventions by great scientists and engineers that humans can be proud of and made a huge impact in the lifestyle of the people. One such great finding of mankind was the internet. Internet has digitalized the world and made convoluted problems easier to solve. Of the many problems solved by internet and with the use of internet, another major hurdle was overcome (i.e.) video streaming. In the early 80 's and 90's when internet was not understood by the common man and it was very difficult to communicate with one another if not in the same place, the invention of video imaging techniques made a breakthrough in making life simpler for the common man. Video streaming has many applications on the internet. The video is consumed by the user while it is being downloaded is the crucial characteristic of video streaming. The major types of video streaming are on-demand and live streaming. Distribution of video packets from the video source to all users before the deadline is the success factor of live video streaming [1].

One of the greatest unfulfilled promise on the internet is live web streaming. Over the last two decades, there have been many technical contributions and great inventions in the field of video streaming, yet there is no single prodigious system that solves all problems of quality service. This effect is due to various problems namely inherent nature of stability and lack of service guarantee, bandwidth instability and stringent requirements of streaming applications [2]. This article reviews the state-of-the-art P2P live video-streaming technologies and their development from a historic perspective. There are several base parameters for exceedingly good user experience such as initial playout latency and video artifacts. Faster initialization is dependent on the rate of throughput. Higher the throughput allows faster initialization of the video buffer [3].

An idea definition of video streaming in real time scenario is the content sent in a compressed format over the web and displayed to the viewer. There are many approaches of peer to peer multimedia streaming. Of the many approaches in peer to peer multimedia streaming, there are two significant and widely used approaches. The first widely used approach is inspired by the swam optimization technique used in the Bit Torrent file sharing application is discussed in [4]. Stream is split into chunks of a fixed size in this swarm optimization technique. The received chunks of a sliding window are maintained by a peer. The second most widely used approach is IP multicast where a peer subscribes to a neighbor to form explicit parent child relationship.

Of the two approaches the swarm-based approach is widely applied in modern day systems. In swarm-based schemes [5-9], at an interval of length $X$ and at the next interval a peer selects the chunks to pull and selects the neighbors to pull these chunks. Selection of chunks by the peer is based on random basis [5], but in most schemes, a peer employs a latest-first or rarest-first policy [6-8]. A peer may select neighbors in several ways to pull the selected chunks are listed as following a). randomly [7], b). based on neighbors' workload [8], c). as per the level of bandwidths and delays to neighbors.

The availability of chunks at peers will determine the propagation trees in the swarm - based approaches [9].

There are two types of tree-based schemes namely network driven [10-13]. Each scheme [10-11] with an objective of lowering the tree cost is actually network driven usually network driven. The combination of swarm and tree-building techniques are best performed in data driven schemes.
Revised Version Manuscript Received on April 12, 2019.

Farooq Sunar Mahammad, School of Computing Science and Engineering, Vellore Institute of Technology (VIT), Vellore, T.N, India

Karthik Balasubramanian, Process and Equipment Engineer, Micron Semiconductor Asia Operations Pte. Ltd., Singapore

T. Sudhakar Babu, Institute of Power Engineering, Department of Electrical Power Engineering, Universiti Tenaga Nasional, Malaysia

V. Madhu iswanatham, School of Computing Science and Engineering, Vellore Institute of Technology (VIT), Vellore, T.N, India 


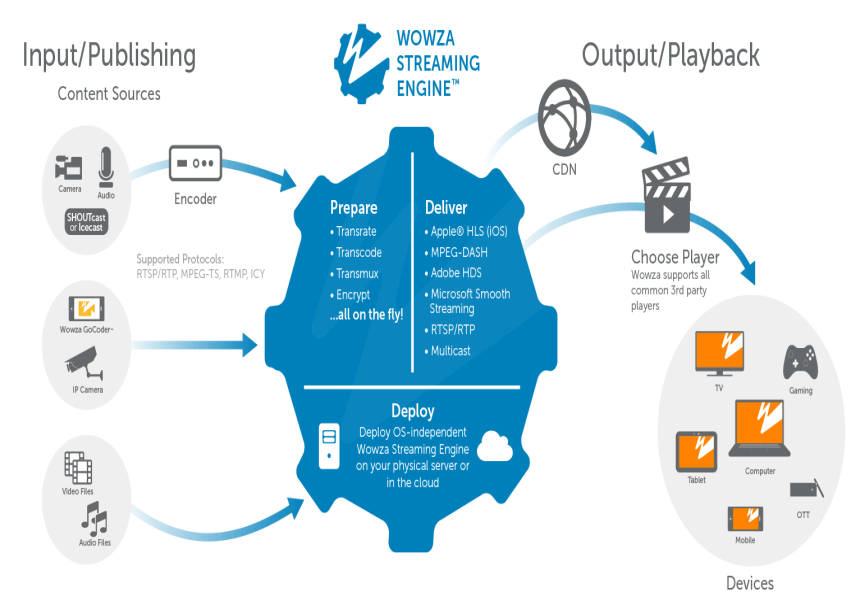

Fig .2.1 Video Streaming Process Flow in Wowza Engine

\section{PEER TO PEER VIDEO STREAMING}

There are many types of video streaming which plays a significant role in our lives. Out of the existing available video imaging techniques, peer to peer video streaming is considered as the most significant and widely used video imaging technique [14]. The client and the server are both the peer. Applications of video streaming are enormous such as sharing of file / data and IP telephony.

The significant steps for peer to peer streaming are as follows [1]:

2. Peer reachability

\subsection{Preliminaries}

Probability distribution decides the peer joining the video channel. The most important objective of the preliminary based approach is that the circulation of video packets to all the peers from the source of video is planned in a such a way that the peers receive all the packets before their respective playback deadlines and each peer receive their respective pack only once during the entire process cycle.

\subsection{Reachability of the peer}

Many peers are not reachable from the outside due to the blockage synchronization of UDP and TCP packets respectively is performed by firewalls and network address translator. An important aspect is that relationships are strongly established as the peer can find sufficient peers and hence this problem is avoided in most research studies.

\section{PROMINENT WORKS ON VIDEO STREAMING IN VARIOUS APPLICATIONS}

There are several publications in the field of video streaming which have contributed to several other domains in a positive way. This section will summarize the prominent works in video streaming in various applications.

The authors in [15] propose a new probabilistic adaptive data replication strategy. The authors have implemented the concept of probabilistic manner and hence calculated the location for replication of data object.

The analysis of e-learning environments and video interaction learning are presented in detail by the authors in [16]. Out of the many different settings understood by the authors, four settings were studied in detail namely interactive video, with non-interactive video and without
1. Preliminaries

video. The results generated proved the value of eLearning and video interaction proved to very successful and enriching.

The authors of [17] have analyzed an important portion of video live streaming. To comprehend the online questions and chat massages online, the authors have implemented the concept of data mining and text mining. The research made the authors understand the inconsistencies as well as resemblances in the understanding of student patterns and participation themes between questions online and chat messages.

The concept of delay playback and client buffer techniques implemented to an online model is developed by the authors in [18]. It is a well-known fact that the server has limited information about the frame sizes of the future and hence the buffer allocation shifts to placing the memory at the server proved by the authors research.

The VCR functionality impacts on network traffic is analyzed by the authors [19]. A state of the art least cost scheme for the effective utilization of MPEG streaming video system to provide full VCR functionality over a network with less requirements is deduced by the authors.

The authors in [20] show the effectiveness of adaptive media playout (AMP). Introduction of limited delay allows the client to buffer less data which shows the variation of the play out speed of media frames contingent on conditions of channels.

An interesting method on low-complexity rate-distortion (R-D) optimized adaptive video streaming based on the concept of rate-distortion hint track (RDHT) is presented by the authors in [21]. The deduced results have proved very effective in comparison to conventional results published in the earlier papers. This gain is achieved with a convoluted process making them appropriate for online R-D optimized streaming.

A pragmatic $\mathrm{P} 2 \mathrm{P}$ - based live video streaming system is discussed inarticle [22]. A study is performed by the authors to comprehend the capacity of service, service quality and duration characteristics.

The significance and application of stable nodes in the $\mathrm{P} 2 \mathrm{P}$ video streaming is detailed in [23]. A set of algorithms for stable node identification and organization is developed and explained in detail.

The authors in [24] present a design overview of the Class $\mathrm{X}$ system of a 3-month pilot deployment at Stanford University. The results demonstrate that the methodology is a practical solution to interactive online lecture viewing.

In [25], the authors present a probability approximation model based on buffer fullness variation on large deviation theory with exemplary and accurate results. The results prove superior compared to earlier approaches.

The benefits of SDN based application aware network is broadly discussed in [26]. The authors determine the overhead caused by each of the presented approaches.

In [27], H.264/AVC video encryption scheme is analyzed where the obtained results show that the proposed scheme is effective in computing. 
The authors in[28] present an analysis on the various algorithms used in video streaming, present their properties and shortcomings.

The authors in [29] have proven results that shoe the proposed scheme can achieve high security at a relatively low compression ratio and bandwidth cost and hence suitable for security multimedia services.

The authors in[30] survey techniques used to further enhance the internet qualityvideo such as network load reduction, network interruption mitigation, and network load distribution.

\section{OPTIMIZATION TECHNIQUES IMPLEMENTED IN VIDEO STREAMING, INDUSTRY PERSPECTIVE}

Video sources are typically divided into two categories: real-time video and non-real-time video.

\subsection{Real-time Video Streaming}

Real time video streaming has much more timing requirements comapred to the other forms video streaming.

\subsection{Non - Real Time Video Streaming}

Non - real time video streaming is a type of streaming that is already prerecorded. Having access to the entire video also allows for digital video recorder. The method can take advantage of many non-real-time video processing optimizations with time shift variations.

\subsection{Industry Perspective} progressively moving towards main stream business. PPlive [31] and SopCast [32] have attracted many downloads and supported several daily online viewers. Fig. 2 shows the timeline of how live video streaming is implemented by Multi-National Companies.

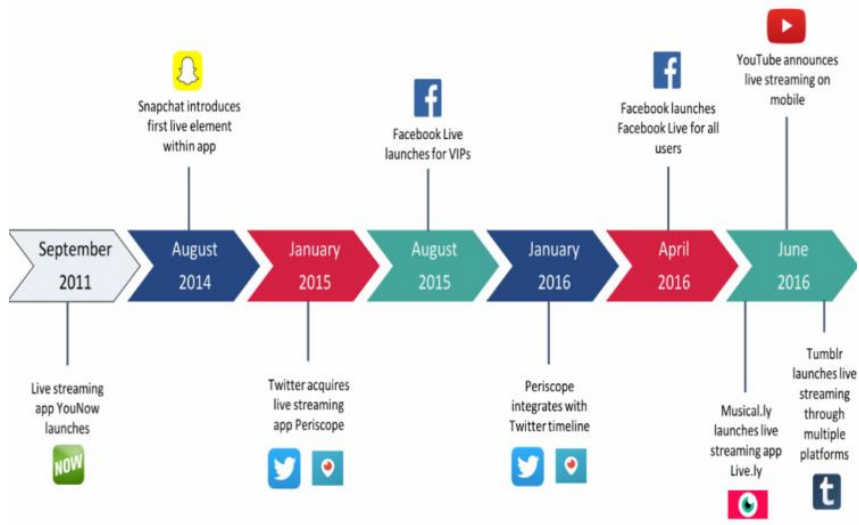

Fig 4.1 Time line of Live video streaming used by Multinational Companies

\section{RESEARCH AREAS IN VIDEO STREAMING \& RESULTS}

\subsection{IP multicast and CDN}

One to many communication paradigms belongs to live streaming. To reduce network traffic, packets need to be duplicated by boxes. An important methodology to be followed by ISPs is to provide IPTV2. [33] provides an IP multicast analyses. To serve nearby users, cache servers are
There is a plethora of streaming services that are

placed tactically by CDNs [34,35].

\subsection{Significant forms of coding in video streaming}

The important forms of coding currently concentrated and focused are the video, channel and network coding. Single and multiple layers are used to encode videos. Reliable and effective transmission are present in single layer coding. Many equal descriptions are encoded in MDC [36,37]. Decoding a receiver using any subset of the descriptions are also performed.

Effective and efficient coding can be obtained by LC compared to single layer coding. The bit rate increases of SVC relative to AVC at the same fidelity can be as low as $10 \%$ [38]. MDC has huge overhead [48] and is rarely used in the realworld. MDC is used in [39-43]; LC is used in $[44,45]$.

\subsection{Network positioning}

ISPs can be single cost information provider as in oracle [46] and P4P [47-49] proposals.

Nearby peers are analyzed in detail from [50-53]. There is an exceptional decrease in cost which is due to the high loss in packets thereby reducing the stability of the system. The authors in [54] proposes that remote peers will contribute to the definite decrease of these effects.

Shorter downloading time play are gifted with peers uploading lot of data. Other incentives include better fidelity of video [55], smoother playback or a shorter playback delay [56]. A game theoretic incentive mechanism is proposed in [57] to dealwith cheating and malicious users.

\subsection{Combination of Electrical and Computer Engineering}

The objective of [58] is to implement a design method of digital parity generator. In [59], performance analysis of hashing method is determined. This method [60] effectively overcomes the problems of geometrical deformation of the target, partial occlusion and allows recovery target leaves the field of view.

\section{CONCLUSION}

In this paper, the most important papers on Video imaging techniques are systematically reviewed. The different works on video imaging techniques are elaborated in detail. Further the article also summarizes the latest techniques in video streaming; it also summarizes the features of all works with respect to video imaging processing techniques. Hence, this paper will be useful to the researchers and new entrants as it is one stop shop for the research of Video Imaging techniques.

\section{REFERENCES}

1. XiangyangZhanget.al. A survey of peer-to-peer live video streaming schemes - An algorithmic perspective, Computer Networks 56 3548-3579(2012)

2. Bo Li. Peer-to-Peer Live Video Streaming on the Internet: Issues, Existing Approaches, and Challenges. IEEE Communications Magazine, June 2007

3. Ma KJ, et al. A survey of schemes for Internet-based video delivery. J Network ComputAppl (2011), doi: 10.1016/j.jnca.2011.02.001

4. <http://www.bittorrent.com>, Accessed Mar. 2011. 
5. V. Pai, K. Kumar, K. Tamilmani, V. Sambamurthy, A.E. Mohr, Chainsaw:eliminating trees from overlay multicast, in: Proceedings of International Workshop on Peer-to-Peer Systems, 2005.

6. X. Zhang, J. Liu, B. Li, T.S.P. Yum, Coolstreaming/donet: a data-driven overlay network for efficient live media streaming, in: Proceedings of IEEE INFOCOM, vol. 3, pp. 13-17, 2005.

7. N. Magharei, R. Rejaie, Prime: peer-to-peer receiver-driven mesh-based streaming, in: Proceedings of IEEE INFOCOM, pp. 1415-1423, 2007

8. F. Pianese, D. Perino, J. Keller, E. Biersack, PULSE: an adaptive incentive-based unstructured P2P live streaming system, IEEE Transactions on Multimedia 9 (8) 1645-1660, (2007).

9. Z. Liu, Y. Shen, K. Ross, S. Panwar, Y. Wang, LayerP2P: using layered video chunks in $\mathrm{p} 2 \mathrm{p}$ live streaming, IEEE Transactions on Multimedia 11 (7) 1340-1352, (2009).

10. Y. Chu, S. Rao, S. Seshan, H. Zhang, A case for end system multicast, IEEE Journal on Selected Areas in Communications 20 (8) 1456-1471, (2002).

11. S. Banerjee, B. Bhattacharjee, C. Kommareddy, Scalable application layer multicast, in: Proceedings of ACM SIGCOMM Conference on Applications Technologies Architectures and Protocols for Computer Communication, vol. 32, pp. 205-217, 2002.

12. F. Wang, Y. Xiong, J. Liu, B. Burnaby, C. Beijing, mTreebone: a hybrid tree/mesh overlay for application-layer live video multicast, in: Proceedings of ICDCS, p. 49, 2007

13. D. Ren, Y.-T. Li, S.-H. Chan, Fast-mesh: a low-delay high-bandwidth mesh for peer-to-peer live streaming, IEEE Transactions on Multimedia 11 (8) 1446-1456, 2009.

14. S. Ali, A. Mathur, H. Zhang, Measurement of commercial peer-to-peer livevideo streaming, in: Proceedings of Workshop in Recent Advances in Peer-to-Peer Streaming, 2006.

15. Neeraj Kumar et. Al. Probabilistic trust aware data replica placement strategy for online video streaming applications in vehicular delay tolerant networks. Mathematical and Computer Modelling 58 3-14, 2013.

16. Dongsong Zhang et. Al. Instructional video in e-learning: Assessing the impact of interactive video on learning effectiveness. Information \& Management 43 15-27, 2006.

17. Wu He. Examining students' online interaction in a live video streaming environment using data mining and text mining.Computers in Human Behavior 29 90-102, 2013.

18. subhabratasenet. Al. Online smoothing of variable-bit-rate streaming video. IEEE transactions on multimedia, vol. 2, no. 1, march 2000

19. chia-wen lin et. Al. Mpeg video streaming with ver functionality, IEEE transactions on circuits and systems for video technology, vol. 11, no. 3, march 2001

20. markkalmanet. Al. Adaptive media playout for low-delay video streaming over error-prone channels, IEEE transactions on circuits and systems for video technology, vol. 14 , no. 6 , june 2004

21. jacobchakareskiet. Al. Rate-distortion hint tracks for adaptive video streaming, IEEE transactions on circuits and systems for video technology, vol. 15, no. 10, october 2005 Video-Streaming Service. IEEE Communications Magazine, June 2007

23. Feng Wang Et. Al. Stable Peers: Existence, Importance, And Application In Peer-To-Peer Live Video Streaming. IEEE Communications Society Subject Matter Experts for Publication In The IEEE INFOCOM 2008 Proceedings.

24. Adityamavlankaret. Al. AN INTERACTIVE REGION-OF-INTEREST VIDEO STREAMING SYSTEM FOR ONLINE LECTURE VIEWING, Proceedings Of 2010 IEEE 18th International Packet Video Workshop

25. Jian Yang Et. Al. Online Buffer Fullness Estimation Aided Adaptive Media Playout For Video Streaming, IEEE TRANSACTIONS ON MULTIMEDIA, VOL. 13, NO. 5, OCTOBER 2011

26. [26] Michael Jarschelet. Al. SDN-Based Application-Aware Networking On TheExample Of YoutubeVideo Streaming, 2013 Second European Workshop On Software Defined Networks

27. Xiaofeng Wang Et. Al. Hash Key-Based Video Encryption Scheme ForH.264/AVC. Signal Processing: Image Communication 25 427-437, 2010

28. Fuwen Liu et. Al. A survey of video encryption algorithms.computers\& security 29 (2010) $3-15$
22. Yun Tang Et. Al. Deploying P2P Networks ForLarge-Scale Live

29. NaeemRamzanet. Al. Video streaming over P2P networks Challenges and opportunities. Signal Processing: Image Communication 27 401-411, 2012.

30. WANG Li-fenget. Al. Perceptual video encryption scheme for mobile application based on H.264. The Journal of China Universities of Posts and Telecommunications, September 2008, 15(Suppl.): 73-78

31. http://www.pplive.com/

32. http://www.sopcast.org/

33. C. Diot, W. Dabbous, J. Crowcroft, Multipoint communication: a survey of protocols, functions, and mechanisms, IEEE Journal on Selected Areas in Communications 15 (3) 277-290, 1997.

34. A. Vakali, G. Pallis, Content delivery networks: status and trends, IEEE Internet Computing 7 (6) 68-74, 2003.

35. J. Dilley, B. Maggs, J. Parikh, H. Prokop, R. Sitaraman, B. Weihl, Globally distributed content delivery, IEEE Internet Computing 50-58, 2002

36. V. Goyal, Multiple description coding: compression meets the network, IEEE Signal Processing Magazine 18 (5) 74-93, 2001.

37. Y. Wang, A. Reibman, S. Lin, Multiple description coding for video delivery, Proceedings of IEEE 93 (1) 57-70, 2005.

38. H. Schwarz, D. Marpe, T. Wiegand, Overview of the scalable video coding extension of the h. 264/avc standard, IEEE Transacions on Circuits and Systems for Video Technology 17 (9) 1103-1120, 2007

39. F. Fitzek, B. Can, R. Prasad, M. Katz, Overhead and quality measurements for multiple description coding for video services, Wireless Personal Multimedia Communications 2 524-528, 2004.

40. V.N. Padmanabhan, H.J. Wang, P.A. Chou, K. Sripanidkulchai, distributing streaming media content using cooperative networking, in: Proceedings of International Workshop onNetwork and Operating Systems Support for Digital Audio and Video, pp. 177-186, 2002.

41. R. Tian, Y. Xiong, Q. Zhang, B. Li, B.Y. Zhao, X. Li, Hybrid overlay structure based on random walks, in: Proceedings of International Workshop on Peer-to-Peer Systems, pp. 1-11, 2005.

42. M. Kobayashi, H. Nakayama, N. Ansari, N. Kato, Robust and efficient stream delivery for application layer multicasting in heterogeneous networks, IEEE Transactions on Multimedia 11 (1) $166-176,2006$

43. N. Magharei, R. Rejaie, Prime: Peer-to-peer receiver-driven mesh-based streaming, in: Proceedings of IEEE INFOCOM, pp. 1415-1423, 2007

44. A. Magnetto, R. Gaeta, M. Grangetto, M. Sereno, TURIN stream a totally push, robust, and efficient P2P video streaming architecture, IEEE Transactions on Multimedia 12 (8) 901-914 2010.

45. [45] Z. Liu, Y. Shen, K. Ross, S. Panwar, Y. Wang, LayerP2P using layered video chunks in $\mathrm{P} 2 \mathrm{P}$ live streaming, IEEE Transactions on Multimedia 11 (7), 1340-1352, 2009.

46. V. Aggarwal, A. Feldmann, C. Scheideler, Can ISPS and P2P users cooperate for improved performance, ACM SIGCOMM Computer Communication Review 37 (3) 40, 2010.

47. H. Xie, Y. Yang, A. Krishnamurthy, Y. Liu, A. Silberschatz, P4P provider portal for $\mathrm{P} 2 \mathrm{P}$ applications, in: Proceedings of ACM SIGCOMM Conference on Data, Communication, 2008, pp. 351-362.

48. D. Choffnes, F. Bustamante, Taming the Torrent: a practical approach to reducing cross-ISP traffic in peer-to-peer systems, 38(4) 363-374, 2008

49. [49] B. Krishnamurthy, J. Wang, On network-aware clustering of web clients, in: Proceedings of ACM SIGCOMM Conference on Applications, Technologies, Architectures, and Protocols for Computer, Communication, pp. 97-110, 2000.

50. T. Ng, H. Zhang, predicting internet network distance with coordinates-based approaches, in Proc, IEEE INFOCOM 170-179, 2002

51. F. Dabek, R. Cox, F. Kaashoek, R. Morris, Vivaldi: a decentralized network coordinate system, ACM SIGCOMM Computer Communication Review 34 (4) 15-26, 2004

52. M. Piatek, H. Madhyastha, J. John, A. Krishnamurthy, T. Anderson, Pitfalls for ISP-friendly P2P design, in: Proceedings of HotNets, pp. 1-6, 2009

Published By:

Blue Eyes Intelligence Engineering 
53. X. Zhang, H. Hassanein, On network utilization of peer-to-peer video live streaming on the Internet, in: IEEE International Conference on Communications (ICC), pp. 1-5, 2011.

54. X. Zhang, H. Hassanein, A neighboring strategy for ISP-friendly peerto- peer video live streaming, in IEEE International Conference on Communications (ICC), pp. 1-5, 2011.

55. Z. Liu, Y. Shen, K. Ross, S. Panwar, Y. Wang, LayerP2P: using layered video chunks in P2P live streaming, IEEE Transactions on Multimedia 11 (7) 1340-1352, 2009.

56. F. Pianese, D. Perino, J. Keller, E. Biersack, PULSE: an adaptive, incentive-based, unstructured P2P live streaming system, IEEE Transactions on Multimedia 9 (8), 1645-1660,2007

57. W. Lin, H. Zhao, K. Liu, Incentive cooperation strategies for peer-topeer live multimedia streaming social networks, IEEE Transactions on Multimedia 11 (3), 396-412, 2009

58. Wahab Musa.Design of Digital Parity Generator Layout Using 0.7-micron Technology.International Journal of Electrical and Computer

Engineering.http://doi.org/10.11591/ijece.v8i6.pp\%25p

59. Performance Analysis of Hashing Methods on the Employment of App. International Journal of Electrical and Computer Engineering. http://doi.org/10.11591/ijece.v8i6.pp\%25p.

60. Khaled Hammemiet. Al. Implementation of Robust Tracking Algorithm on Nano-Computer.International Journal of Electrical and Computer Engineering. http://doi.org/10.11591/ijece.v8i6.pp\%25p.

\section{AUTHORS PROFILE}

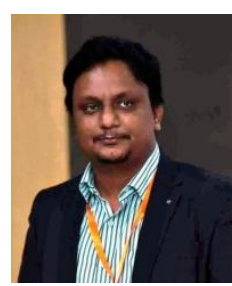

Mr. Farooq Sunar Mahammad is a Research Scholar in the School of Computing Science and Engineering, VIT University, Vellore, India. He received his BTech in Information Technology from the JNT University, Anantapuramu, India and MTech in Computer Science and Engineering from the JNT University Hyderabad, India. Acting as a Editorial Board Member and Reviewer for various reputed Journals. He has several years of experience working in academia, teaching and research. His research interests include video streaming, high performance computing, and information security.

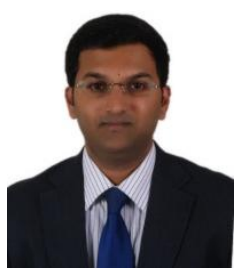

Mr. Karthik Balasubramanian is currently working as a Process and Equipment Engineer at Micron Semiconductor Asia Operations Private Limited, Singapore. He completed his Master of Science (Power Engineering) at Nanyang Technological University (NTU), Singapore. Prior to pursuing his master's degree, he was working as an Electrical, Instrumentation Control and Telecommunication Engineer at Saipem, Chennai, India from July 2014 to May 2017. He completed his B. Tech in Electrical and Electronics Engineering with Minor in Industrial Automation at Vellore Institute of Technology (VIT), Vellore, India, in 2014. He has published many research papers in highly reputed national and international journals. He has served as reviewer for various reputed national and international journals.

Mr. Karthik Balasubramanian was the president of Toastmasters International - VIT University Chapter. He has received multiple High-Performance leadership awards from Toastmasters International, USA. His research interests are Fuel cell system design, PV system design, Semiconductors, Power Systems and applications of Power electronics in Renewable Energy systems.

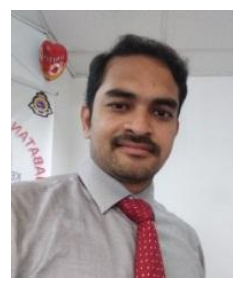

Dr. T SudhakarBabu received Ph.D from VIT University, Vellore, India, in 2017. He is currently working as a researcher in Institute of Power Engineering, Dep, of Electrical Power Engineering, Universiti Tenaga Nasional (UNITEN), Malaysia. He has published more than 30 research articles in various reputed international journals. Acting as editorial board members and reviewers for various reputed journals like IEEE, IEEE Access, IET, Elsevier and Taylor and Francis. His areas of interest are design and implementation of solar PV systems, Renewable energy resources, power management for hybrid energy systems, fuel cell technologies, electric vehicle and smart grid.

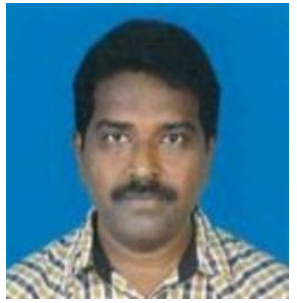

Dr. V. Madhu Viswanatham is an Associate Professor in the School of Computing Science and Engineering, VIT University, Vellore, India. He received his $\mathrm{PhD}$ in Computer Science and Technology from the SK University, Anatapur, India. His research interests are in the areas of information security, wireless networks, grid computing and cloud computing. He has several years of experience working in academia, teaching and research 